\title{
A Comprehensive Analysis of Novel Disulfide Bond Introduction Site into the Constant Domain of Human Fab
}

Hitomi Nakamura

Sojo University

Moeka Yoshikawa

Sojo University

Naoko Oda-Ueda

Sojo University

Tadashi Ueda

Kyushu University

Takatoshi Ohkuri ( $\nabla$ ohkuri@ph.sojo-u.ac.jp )

Sojo University

\section{Research Article}

Keywords: intermolecular disulfide bond, conformational protein stability, Fab's , herapeutic IgG, MOE software,molecular simulator

Posted Date: March 17th, 2021

DOl: https://doi.org/10.21203/rs.3.rs-294111/v1

License: (c) (i) This work is licensed under a Creative Commons Attribution 4.0 International License. Read Full License 
A comprehensive analysis of novel disulfide bond introduction site into the constant domain of human Fab

Hitomi Nakamura ${ }^{1}$, Moeka Yoshikawa ${ }^{1}$, Naoko Oda-Ueda ${ }^{1}$, Tadashi Ueda ${ }^{2}$, Takatoshi Ohkuri ${ }^{1 *}$.

${ }^{1}$ Faculty of Pharmaceutical Sciences, Sojo University

${ }^{2}$ Graduate School of Pharmaceutical Sciences, Kyushu University

*Corresponding author

Takatoshi Ohkuri

Faculty of Pharmaceutical Sciences, Sojo University, 4-22-1 Ikeda, Nishikuku, Kumamoto 860-0082, Japan

E-mail address: ohkuri@ph.sojo-u.ac.jp 


\section{(ABSTRACT)}

Generally, intermolecular disulfide bond contribute to the conformational protein stability. To identify sites where intermolecular disulfide bonds can be introduced into the Fab's constant domain of the therapeutic IgG, Fab mutants were predicted using the MOE software, a molecular simulator, and expressed in Pichia pastoris. SDS-PAGE analysis of the prepared Fab mutants from $P$. pastoris indicated that among the nine analyzed Fab mutants, the H: F130C-L: Q124C, H: F174C-L: S176C, H: V177C-L: Q160C, H: F174C-L: S162C, H: F130C-L: S121C, and H: A145C-L: F116C mutants mostly formed intermolecular disulfide bonds. All these mutants showed increased thermal stabilities compared to those without intermolecular disulfide bonds. In the other mutants, the intermolecular disulfide bond could not be completely formed, and the L132C-F118C mutant showed only a slight decrease in binding activity and $\beta$-helix content, owing to adverse intermolecular disulfide bond effects. Thus, our comprehensive analysis reveals the introduction of intermolecular disulfide bonds in the Fab's constant domain is possible at various locations. These findings provide important insights for accomplishing human Fab stabilization. 


\section{(Introduction)}

Monoclonal antibodies are highly effective against intractable diseases, such as cancer and rheumatism. ${ }^{1-3}$ While the IgG molecule has several advantages, it must be produced in mammalian cell lines which require superior technology, resulting in high costs associated with the production of the necessary therapeutic doses. Alternatively, antibody fragments, in particular the single-chain antibody fragment $(\mathrm{scFv})$ and fragment antigen binding (Fab) can be efficiently expressed in microbial expression systems. Since $\mathrm{Fab}$ is more stable than $\mathrm{scFv}$, it is therefore more suitable for clinical applications, ${ }^{4}$ such as certolizumab pegol, which is used for the treatment of rheumatoid arthritis and Crohn's disease. ${ }^{5}$

However, therapeutic proteins are at risk of degradation during the purification process or product storage, leading to protein aggregation or the appearance of antigenicity. ${ }^{6-10}$ For instance, deamidation of the asparagine residues is one of the most frequent modifications, which might cause changes in bioactivity, bioavailability, or immunogenicity. ${ }^{11-18}$ Furthermore, it has been shown that the deamidation of Asn 30 in the light chain of a recombinant $\operatorname{IgG}$ reduces its binding potency to $70 \% .{ }^{19}$ While proteins usually maintain an equilibrium between the native and denatured states, the equilibrium greatly favors the native state under physiological conditions. If proteins are inherently unstable, the rate of transition from the native to the denatured state is relatively rapid, resulting in protein degradation, ${ }^{20-22}$ therefore, the stabilization of therapeutic proteins is effective in suppressing protein degradation. Intermolecular disulfide bond generally contribute to 
the conformational protein stability. A previous study has shown that the introduction of intermolecular disulfide bonds increased the conformational stability of dimer proteins. ${ }^{23-26}$ However, few attempts have been made to introduce novel intermolecular disulfide bonds into Fab to increase protein stability.

An expression system of IgG1's Fab developed as the therapeutic antibody adalimumab, anti-human TNF- $\alpha$, was constructed in the yeast strain $P$. pastoris. We targeted Fab's constant domain to promote the applicability of our findings to other human Fabs. Furthermore, we predicted the intermolecular disulfide bond mutation sites of adalimumab Fab using the Disulfide Scan modeling software, and further analyzed the first mutants, the Fab-H (H:V177C) and L chain (L:Q160C), to be successfully expressed in yeast. ${ }^{27}$ The Fab mutant was confirmed to undergo thermal stabilization through the formation of an intermolecular disulfide bond. In this study, in order to identify the further sites that allow the introduction of intermolecular disulfide bonds in the adalimumab Fab's constant domain for improving the stability, other presumed mutants with engineered intermolecular disulfide bonds were prepared from $P$. pastoris and collected information on their stabilizations.

\section{Results}

\section{Preparation of intermolecular SS mutants.}

To introduce an intermolecular disulfide bond in the Fab's constant domain, the prediction of the formation of disulfide bond by mutation was performed 
using the Disulfide Scan modeling software, and the structure of adalimumab Fab (protein data bank number : 4NYL) was used as a modeling template. The mutation sites of the prepared mutants (intermolecular SS mutants: Mut1-Mut9) are shown in Table 1. The wild-type Fab has one intermolecular disulfide bond at the $\mathrm{C}$-terminal end. To facilitate the analysis of intermolecular disulfide bond formation, mutations were made to Fab in which the cysteine residues to form intact $\mathrm{C}$-terminal intermolecular disulfide bonds (H:Cys224-L:Cys214) in human Fab were replaced with alanine $(\Delta \mathrm{SSWT})$; resulting that we can detect quickly whether or not an intermolecular disulfide bond in mutant Fabs form using SDS-PAGE of respective mutant treated in the absence or presence of reductant. The intermolecular SS mutants (Mut1-Mut9) were expressed in the yeast strain $P$. pastoris and purified as previous methods. ${ }^{28}$ The SDS-PAGE of intermolecular SS mutants after the final purification step is shown in Figure 1. Under non-reducing conditions, the wild-type Fab (WT), which is connected at the C-terminal by the disulfide bonds of the Fab-H (theoretical value $23.8 \mathrm{kDa}$ ) and $\mathrm{L}$ chain (theoretical value $23.4 \mathrm{kDa}$ ) showed a single protein band at approximately $47-50 \mathrm{kDa}$, while $\Delta \mathrm{SSWT}$ showed two protein bands derived from the Fab-H and $\mathrm{L}$ chains. Further, the intermolecular SS mutants were evaluated for disulfide bond formation based on the results obtained for WT and $\Delta$ SSWT (Fig. 1a). Mut1 contained disulfide and non-disulfide formations, while Mut2 did not contain disulfide formations. Similarly, Mut9 exhibited a small number of disulfide forms. In contrast, Mut3, Mut4, Mut5, Mut6, Mut7, and Mut8 mostly formed 
intermolecular disulfide bonds. Since the same results were obtained under reducing conditions, it was confirmed that the protein bands of Fab-H and $\mathrm{L}$ chains were present in a 1:1 ratio on all intermolecular SS mutants (Fig. 1b).

\section{Characterization of intermolecular SS mutants.}

The antigen-binding activity of intermolecular SS mutants was examined using ELISA (Fig. 2). While there was no difference in the $450 \mathrm{~nm}$ signal increase depending on the Fab concentration among all mutants, Mut9 showed a slight decrease in activity. The CD spectra of the intermolecular SS mutants were recorded in $50 \mathrm{mM} \mathrm{KH}_{2} \mathrm{PO}_{4}$ buffer, $\mathrm{pH} 6.5$ (Fig. 3). The spectrum of Mut9 showed a slight decrease in $\beta$-helix content compared to the other mutants. From these results, we concluded that the intermolecular disulfide bond introduction had almost no effect on the antigen-binding activity and structure of Fab, except for Mut9 wherein a slight effect was observed.

\section{Thermostability of intermolecular SS mutants.}

To investigate the contribution of the introduced intermolecular disulfide bond to thermostability, Mut3, Mut4, Mut5, Mut6, Mut7, and Mut8 were measured using DSC (Fig. 4). A previous study indicated that the melting temperature (Tm) values for $\Delta$ SSWT and WT were $69.9^{\circ} \mathrm{C}$ and $74.9^{\circ} \mathrm{C}$, respectively. ${ }^{27}$ The Tm values for intermolecular SS mutants, Mut3, Mut4, Mut6, Mut7, and Mut8 were higher than that of $\Delta$ SSWT, indicating that the 
introduction of intermolecular disulfide bonds improved the Fab's thermostability, as previously reported for Mut5.

\section{Refolding of WT and Mut1.}

Refolding of WT and Mut 1 from the reduced and denatured condition were performed using the stepwise dialysis method as previously described ${ }^{29}$ with slight modifications. The formation of intermolecular disulfide bonds of refolded WT and Mut1 was analyzed using SDS-PAGE under non-reduced conditions (Fig. 5). Refolded WT showed a single band at the position corresponding to the Fab that formed the intermolecular disulfide bond. Conversely, refolded Mut1 contained disulfide and non-disulfide forms similar to those expressed in $P$. pastoris.

\section{(Discussion)}

In a previous study, it was observed that mutations in the adalimumab's Fab-H chain (H:V177C) and L chain (L:Q160C) succeeded in introducing intermolecular disulfide bond in the constant domain (corresponding to Mut5). ${ }^{27}$ Screening of numerous yeast expression strains allowed Mut1, Mut2, Mut3, Mut4, Mut6, Mut7, Mut8, and Mut9 to be expressed and purified from $P$. pastoris. The mutation sites of the intermolecular SS mutants were located at the buried interface of the $\mathrm{CH} 1$ and $\mathrm{CL}$ domains (Fig. 6). In line with the phenotype observed in Mut5, the SDS-PAGE analysis of these intermolecular SS mutants revealed that Mut3, Mut4, Mut6, Mut7, and Mut8 formed mostly intermolecular disulfide bonds (Fig. 1). Mut4, Mut5, 
and Mut6 are located near the top of the constant domain, while Mut3, Mut7, and Mut8 are near the bottom (Fig. 6). The SDS-PAGE performed under non-reducing conditions revealed band differences between Mut3, Mut7, and Mut8 and WT or Mut4, Mut5, and Mut6, which might be explained by the different disulfide bond positions. DSC analysis indicated that the Tm values of $\triangle \mathrm{SSWT}$ obtained following thermal denaturation were $5{ }^{\circ} \mathrm{C}$ lower than those of WT, indicating that the intermolecular disulfide bond has significantly contributed to the thermal stability of Fab. ${ }^{27}$ Therefore, intermolecular SS mutants that formed mostly intermolecular disulfide bonds were further analyzed using DSC (Fig. 4). Mut3, Mut4, Mut5, Mut6, Mut7, and Mut8 showed higher Tm values than $\triangle$ SSWT $\left(\mathrm{Tm}: 69.9^{\circ} \mathrm{C}\right)$, proving that the formation of intermolecular disulfide bonds increases Fab's thermal stability.

Tm values of Mut3, Mut4, Mut6, Mut7, and Mut8 were slightly lower than that of Mut5, which was equivalent to that of WT. Several hydrophobic amino acids have been reported to be located at the interface of the $\mathrm{CH} 1$ and CL domains and play an important role in molecular interactions. ${ }^{30,31}$ Since the mutations of Mut3, Mut4, Mut6, Mut7, and Mut8 include the substitution of phenylalanine (a highly hydrophobic amino acid) as H:F130C, H:F174C, L:F116C, it is possible that Fab's thermostability was hindered by this substitution. Mut9, which exhibits insufficient intermolecular disulfide bond formation, is characterized by mutations of hydrophobic amino acids to cysteine in both Fab-H and L chain. Therefore, Mut9 seems to have a slight effect not only on disulfide bond formation, but also on antigen binding and 
secondary structure (Fig. 2 and 3). Conversely, Mut1 and Mut2 showed insufficient intermolecular disulfide bond formation, although the highly hydrophobic amino acids were not substituted. To verify the possibility that the mutation site of cysteine was modified in yeast cells with a substance such as glutathione, Mut1 was refolded from reductive denaturation conditions. While the SDS-PAGE analysis of refolded Mut1 showed that it did not completely form intermolecular disulfide bonds, the WT mostly formed intermolecular disulfide bonds under identical conditions (Fig. 5). Thus, we hypothesize that Mut1 might be unable to form intermolecular disulfide bonds due to the influence of the surrounding environment.

The C-terminal intermolecular disulfide bond in the constant domain plays an important role in Fab's stability.,27 While there are few reports on introduction of intermolecular disulfide bonds at other positions in the Fab's constant domain, in this study, we showed that intermolecular disulfide bonds can be introduced at various positions in the human Fab's constant domain and they might contribute to its stability. In the study so far, the cysteine residues to form intact $\mathrm{C}$-terminal intermolecular disulfide bonds (H:Cys224-LCys214) in human Fab was utilized to link to polyethyleneglycol, the resulting Fab has a superior pharmacokinetics in mice. ${ }^{32,33}$ Thus, the present study on generation of disulfide bonds in human Fab would be useful in developing novel Fab therapeutics.

\section{Methods}

\section{Design of intermolecular disulfide bond}


A pair of mutations capable of forming disulfide bonds in the constant domain between Fab-H chain and $\mathrm{L}$ chain were identified using the "Disulfide Scan" modeling software in the Molecular Operating Environment platform (MOE; MOLSIS inc., Japan). The structure of adalimumab Fab (protein data bank number : 4NYL) was used as a modeling template.

\section{Construction of adalimumab Fab expression plasmid}

Each expression cassette of the Fab-H chain and L chain genes was both inserted into the expression vector pPICZ $\alpha$ A (Invitrogen, USA) and constructed as a co-expression plasmid according to previous study. ${ }^{27}$ Sitedirected mutagenesis for Fab gene were performed by PCR using mutagenic primer. ${ }^{34}$ Each Fab-H and $\mathrm{L}$ chain gene was designed to attach an extra serine residue at the $\mathrm{N}$-terminus for efficient processing of $\alpha$-factor signal peptide. ${ }^{28}$

\section{Screening of high-level expression strains of intermolecular SS mutants}

Yeast $P$. pastoris $(\mathrm{X}-33)$ was transformed by electroporation using a linearized co-expression plasmid by digestion with PmeI according manufacture protocol (Invitrogen, USA). The transformant cells were plated on YPDS medium containing $100 \mu \mathrm{g} / \mathrm{ml}$ of zeocin. The YPDS plate incubated at $30^{\circ} \mathrm{C}$ for 3 days and a number of the larger colonies that grew on the plate were selected. The selected colonies were each inoculated in 2 $\mathrm{ml}$ of BMGY medium at $30^{\circ} \mathrm{C}$ for 3 days with shaking. After growth culture was centrifuged at $3,000 \times \mathrm{g}$ for $7 \mathrm{~min}$, the pellet was resuspended in $2 \mathrm{ml}$ of 
BMMY medium and incubated at $30^{\circ} \mathrm{C}$ with shaking. To induce expression of Fab mutants, methanol was added to the BMMY medium at final concentration of $0.1 \%$ every 24 hours. After 4 days, the each culture supernatant was harvested by centrifugation at $8,000 \times \mathrm{g}$ for $7 \mathrm{~min}$. Using the culture supernatant, the strain having the highest binding activity to TNF $\alpha$ by ELISA was selected as the highly expression strain of adalimumab Fab.

\section{Large scale expression and purification of intermolecular SS mutants}

The selected expression strain were grown in $400 \mathrm{~mL}$ of BMGY medium and intermolecular SS mutants were expressed in $400 \mathrm{~mL}$ BMMY medium according to previous study. ${ }^{27}$ The expressed Fab was purified by a slight modification of the previous methods. ${ }^{27}$ Namely, the culture supernatant was brought to $60 \%$ ammonium sulfate saturation and removed the residual medium by centrifugation at $10,000 \times \mathrm{g}$ for $15 \mathrm{~min}$ at $4^{\circ} \mathrm{C}$. The precipitation of protein was resuspended in $50 \mathrm{mM} \mathrm{CH}{ }_{3} \mathrm{COOH}-\mathrm{CH}_{3} \mathrm{COONa}$ buffer, $\mathrm{pH} 4$. The solution after dialysis against same buffer was then bound to a SP Toyopearl 650M column $(2.2 \mathrm{~cm} \times 12 \mathrm{~cm}$; Tosoh, Japan $)$ equilibrated with $50 \mathrm{mM} \mathrm{CH}_{3} \mathrm{COOH}-\mathrm{CH}_{3} \mathrm{COONa}$ buffer, $\mathrm{pH} 4.0$, and eluted with $1.0 \mathrm{M} \mathrm{NaCl}$ in the same buffer. The pooled protein was dialyzed against $50 \mathrm{mM}$ $\mathrm{CH}_{3} \mathrm{COOH}-\mathrm{CH}_{3} \mathrm{COONa}$ buffer, $\mathrm{pH} 5.0$ and was loaded onto a Blue Sepharose 6 Fast Flow column $(1.5 \mathrm{~cm} \times 6 \mathrm{~cm}$; GE Healthcare, USA $)$ and equilibrated with $50 \mathrm{mM} \mathrm{CH} \mathrm{CH}_{3} \mathrm{COOH}-\mathrm{CH}_{3} \mathrm{COONa}$ buffer, $\mathrm{pH}$ 5.0. The protein was eluted with $0.4 \mathrm{M} \mathrm{NaCl}$ in the same buffer. The eluted fraction 
containing Fab was dialyzed against $50 \mathrm{mM} \mathrm{CH}_{3} \mathrm{COOH}-\mathrm{CH}_{3} \mathrm{COONa}$ buffer, $\mathrm{pH} 5.0$ and further purified by cation exchange chromatography using a resource S column (column volume was $1 \mathrm{ml}$; GE Healthcare, USA) with AKTA purifier system. The column was equilibrated with $50 \mathrm{mM}$ $\mathrm{CH}_{3} \mathrm{COOH}-\mathrm{CH}_{3} \mathrm{COONa}$ buffer, $\mathrm{pH}$ 5.0, and eluted with a $30 \mathrm{ml}$ linear gradient of $\mathrm{NaCl}$ from $0-0.4 \mathrm{M}$ in the same buffer.

\section{Antigen-binding activities of intermolecular SS mutants}

The antigen-binding activities of intermolecular SS mutants for human TNF $\alpha$ were determined by ELISA as described previously. ${ }^{27}$ The recombinant human $\mathrm{TNF} \alpha$ was prepared using Escherichia coli expression systems. ${ }^{27}$ The plate (F96 Nunc-immunoplate; Thermo Scientific, USA) was coated with $\mathrm{TNF} \alpha(0.2 \mu \mathrm{M})$ in $50 \mathrm{mM} \mathrm{NaHCO} 3$ buffer, pH 9.6, at $4^{\circ} \mathrm{C}$ overnight. The wells on plate were washed with $20 \mathrm{mM}$ Tris-HCl, $\mathrm{pH}$ 7.6, containing $0.14 \mathrm{M} \mathrm{NaCl}$ and $0.05 \%$ Tween 20 (washing buffer) and then blocked for 60 min at RT with washing buffer containing $2 \%$ skim milk (blocking buffer). After the washing steps, the solutions containing intermolecular SS mutants were added (50 $\mu 1 /$ well) and incubated for $60 \mathrm{~min}$ at RT. After washing step, the incubated with anti-human Fab conjugated with horseradish peroxidase polyclonal antibody (Abliance, France) for 60 min at RT. After washing step, substrate solution for peroxidase (ELISA POD Substrate TMB Solution Easy; Nacalai Tesque, Japan) was added (100 $\mu 1 /$ well) and incubated at RT. The reaction was terminated by the addition of $1 \mathrm{M} \mathrm{H}_{2} \mathrm{SO}_{4}(100 \mu \mathrm{l} /$ well). The absorbance was measured at $450 \mathrm{~nm}$ using 
microplate spectrometer (Microplate Photometer Multiskan FC; Thermo Scientific, USA).

\section{CD spectra of intermolecular SS mutants}

The intermolecular SS mutants were prepared at a concentration of 0.2 $\mathrm{mg} / \mathrm{ml}$ in $50 \mathrm{mM} \mathrm{KH}{ }_{2} \mathrm{PO}_{4}$ buffer, $\mathrm{pH}$ 6.5. Then, the $\mathrm{CD}$ spectra were collected on a J720-spectropolarimeter (Jasco, Japan).

\section{Differential scanning calorimetry (DSC) measurements}

DSC measurements were performed using a Nano DSC (TA-instrument, USA) as described previously. ${ }^{27}$ Thermograms of intermolecular SS mutants were monitored from 25 and $90{ }^{\circ} \mathrm{C}$ at a scan rate of $60^{\circ} \mathrm{C} / \mathrm{h}$. The protein solutions were prepared at concentration of $0.2 \mathrm{mg} / \mathrm{ml}$ in $50 \mathrm{mM} \mathrm{KH}{ }_{2} \mathrm{PO}_{4}$ buffer, $\mathrm{pH}$ 6.5. The data was analyzed to obtain the temperature $\left(T_{\mathrm{m}}\right)$ using Nanoanalyze software (TA-instrument, USA).

\section{Refolding of the wild-type and Fab mutants}

Refolding of WT and Mut1 were performed using the stepwise dialysis method as previously described ${ }^{29}$ with slight modifications. Briefly, the lyophilized WT and Mut1 were dissolved in $0.1 \mathrm{M}$ Tris- $\mathrm{HCl}$ buffer, $1 \mathrm{mM}$ EDTA, pH 8.0 containing $8 \mathrm{M}$ urea and reduced with cysteamine (final concentration of $30 \mathrm{mM}$ ) at $40^{\circ} \mathrm{C}$ for $90 \mathrm{~min}$. After denaturation and reduction, 
cystamine was added to the reduced solution at final concentration of $10 \mathrm{mM}$ (redox solution) and incubated at $40^{\circ} \mathrm{C}$ for $20 \mathrm{~min}$. The redox solution was dialyzed against $0.1 \mathrm{M}$ Tris- $\mathrm{HCl}$ buffer, $1 \mathrm{mM}$ EDTA, $0.3 \mathrm{mM}$ cysteamine, $0.1 \mathrm{mM}$ cystamine, $\mathrm{pH} 8.0$ (dialysis buffer) containing $4 \mathrm{M}$ urea at $4^{\circ} \mathrm{C}$ for 4h. Then, the stepwise dialysis was carried out for dialysis buffer containing $2 \mathrm{M}, 1 \mathrm{M}$, and $0 \mathrm{M}$ urea every 12 hours. After final stepwise dialysis, further dialysis was performed against $0.1 \mathrm{M}$ Tris- $\mathrm{HCl}$ buffer, $1 \mathrm{mM}$ EDTA, $\mathrm{pH} 8.0$ in the absence of cysteamine and cystamine. 


\section{References}

1. Plückthun, A. \& Skerra, A. Expression of functional antibody Fv and Fab fragments in Escherichia coli. Methods Enzymol. 178, 497-515 (1989).

2. King, D.J. et al. Expression, purification and characterization of a mouse-human chimeric antibody and chimeric Fab' fragment. Biochem. J. 15, 317-323 (1992).

3. Sun, C., Wirsching, P. \& Janda, K. D. Enabling ScFvs as multi-drug carriers: a dendritic approach. Bioorg. Med. Chem. 11, 1761-1768 (2003).

4. Röthlisberger, D., Honegger, A. \& Plückthun, A. Domain interactions in the Fab fragment: a comparative evaluation of the single-chain Fv and Fab format engineered with variable domains of different stability. J. Mol. Biol. 347, 773-789 (2005).

5. Goel, N. \& Stephens, S. Certolizumab pegol. MAbs. 2, 137-147 (2010).

6. Cromwell, M.E., Hilario, E. \& Jacobson, F. Protein aggregation and bioprocessing. AAPS. J. 8, E572-529 (2006).

7. Shire, S. J. Formulation and manufacturability of biologics. Curr. Opin. Biotechnol. 20, 708-714 (2009).

8. Vázquez-Rey, M. \& Lang, D. A. Aggregates in monoclonal antibody manufacturing processes. Biotechnol. Bioeng. 108, 1494-1508 (2011).

9. Joshi, V., Shivach, T., Kumar, V., Yadav, N. \& Rathore, A. Avoiding antibody aggregation during processing: establishing hold times. Biotechnol. J. 9, 1195-1205 (2014). 
10. Torisu, T., Maruno, T., Hamaji, Y., Ohkubo, T. \& Uchiyama, S. Synergistic Effect of Cavitation and Agitation on Protein Aggregation. J. Pharm. Sci. 106, 521-529 (2017).

11. Geiger, T. \& Clarke, S. Deamidation, isomerization, and racemization at asparaginyl and aspartyl residues in peptides. Succinimide-linked reactions that contribute to protein degradation. J. Biol. Chem. 262, 785-794 (1987).

12. Brange, J., Langkjaer, L., Havelund, S. \& Vølund, A. Chemical stability of insulin. 1. Hydrolytic degradation during storage of pharmaceutical preparations. Pharm. Res. 9, 715-726 (1992).

13. Tomizawa, H., Yamada, H. \& Imoto, T. The mechanism of irreversible inactivation of lysozyme at pH 4 and 100 degrees C. Biochemistry. 33, 13032-13037 (1994). doi: 10.1021/bi00248a012.

14. Kosky, A. A., Razzaq, U. O., Treuheit, M. J. \& Brems, D. N. The effects of alphahelix on the stability of Asn residues: deamidation rates in peptides of varying helicity. Protein. Sci. 8, 2519-2523 (1999).

15. Fujii, N., Matsumoto, S., Hiroki, K. \& Takemoto, L. Inversion and isomerization of Asp-58 residue in human alphaA-crystallin from normal aged lenses and cataractous lenses. Biochim. Biophys. Acta. 1549, 179-187 (2001).

16. Robinson, N. E. \& Robinson, A. B. Deamidation of human proteins. Proc. Natl. Acad. Sci. USA. 98, 12409-12413 (2001).

17. Liu, H., Gaza-Bulseco, G., Faldu, D., Chumsae, C.\& Sun, J. Heterogeneity of monoclonal antibodies. J. Pharm. Sci. 97, 2426-2447 (2008).

18. Luo, Q. et. al. Chemical modifications in therapeutic protein aggregates generated under different stress conditions. J. Biol. Chem. 286, 25134-25144 (2011). 
19. Harris, R. J. et. al. Identification of multiple sources of charge heterogeneity in a recombinant antibody. J. Chromatogr. B. Biomed. Sci. Appl. 752, 233-245 (2001).

20. Careri, G., Fasella, P. \& Gratton, E. Statistical time events in enzymes: a physical assessment. CRC. Crit. Rev. Biochem. 3, 141-164 (1975).

21. Cooper, A. Protein fluctuations and the thermodynamic uncertainty principle. Prog. Biophys. Mol. Biol. 44, 181-214 (1984).

22. Závodszky, P., Kardos, J. \& Svingor Petsko, G. A. Adjustment of conformational flexibility is a key event in the thermal adaptation of proteins. Proc. Natl. Acad. Sci. USA. 95, 7406-7411 (1998).

23. Gokhale, R.S., Agarwalla, S., Francis, V. S., Santi, D. V. \& Balaram, P. Thermal stabilization of thymidylate synthase by engineering two disulfide bridges across the dimer interface. J. Mol. Biol. 235, 89-94 (1994).

24. Velanker, S. S. et al. Disulfide engineering at the dimer interface of Lactobacillus casei thymidylate synthase: crystal structure of the T155C/E188C/C244T mutant. Protein. Sci. 8, 930-933 (1999).

25. Bunting, K. A., Cooper, J.B., Tickle, I. J. \& Young, D. B. Engineering of an intersubunit disulfide bridge in the iron-superoxide dismutase of Mycobacterium tuberculosis. Arch. Biochem. Biophys. 397, 69-76 (2002).

26. Ohkuri, T. et al. A protein's conformational stability is an immunologically dominant factor: evidence that free-energy barriers for protein unfolding limit the immunogenicity of foreign proteins. J. Immunol. 185, 4199-4205 (2010).

27. Nakamura, H., Oda-Ueda, N., Ueda, T. \& Ohkuri, T. A novel engineered interchain disulfide bond in the constant region enhances the thermostability of adalimumab Fab. Biochem. Biophys. Res. Commun. 495, 7-11 (2018). 
28. Ohkuri, T., Murase, E., Sun, S. L., Sugitani, J. and Ueda, T. Characterization of deamidation at Asn138 in L-chain of recombinant humanized Fab expressed from Pichia pastoris. J. Biochem. 154, 333-340 (2013).

29. Fujii, T., Ohkuri, T., Onodera, R. and Ueda, T. Stable supply of large amounts of human Fab from the inclusion bodies in E. coli. J. Biochem. 141, 699-707 (2007).

30. Carredano, E. et al. A novel and conserved pocket of human kappa-Fab fragments: design, synthesis, and verification of directed affinity ligands. Protein. Sci. 13, 1476-1488 (2004).

31. Teerinen, T., Valjakka, J., Rouvinen, J. \& Takkinen, K. Structure-based stability engineering of the mouse IgG1 Fab fragment by modifying constant domains. $J$. Mol. Biol. 361, 687-697 (2006).

32. Khalili, H., Godwin, A., Choi, J. W., Lever, R. \& Brocchini, S. Comparative binding of disulfide-bridged PEG-Fabs. Bioconjug. Chem. 23, 2262-2277 (2012).

33. Nakamura, H., Anraku, M., Oda-Ueda, N., Ueda, T. \& Ohkuri, T. C-Terminal Cysteine PEGylation of Adalimumab Fab with an Engineered Interchain SS Bond. Biol. Pharm. Bull. 43, 418-423 (2020).

34. Mine, S., Ueda, T., Hashimoto, Y., Tanaka, Y. and Imoto, T. High-level expression of uniformly $15 \mathrm{~N}$-labeled hen lysozyme in Pichia pastoris and identification of the site in hen lysozyme where phosphate ion binds using NMR measurements. FEBS. Lett. 448, 33-37 (1999). 


\section{Acknowledgements}

We would like to thank Editage (www.editage.jp) for English language editing. This study was supported by JSPS KAKENHI (16K08922). 
(Figure legends)

Figure 1. SDS-PAGE analysis of purified intermolecular SS mutants. The samples were analyzed by $12 \%$ SDS-PAGE under (a) non-reduced condition and (b) reduced condition. Lane M: protein markers. Lane 1: WT, Lane 2: $\triangle$ SSWT, Lane 3: Mut1, Lane 4: Mut2, Lane 5: Mut3, Lane 6: Mut4, Lane 7: Mut5, Lane 8: Mut6, Lane 9: Mut7, Lane 10: Mut8, Lane 11: Mut9. The gels were stained with SimplyBlue safe stain (Invitrogen) and were then imaged using iBright FL1000 (Thermo Fisher Scientific).

Figure 2. The antigen-binding activity of intermolecular SS mutants. The ELISA plate was coated with TNF $\alpha$, followed by addition of intermolecular SS mutants at various concentrations. Anti-human Fab conjugated with horseradish peroxidase polyclonal antibody was used for evaluating the antigen-binding activity. The absorbance was measured at $450 \mathrm{~nm}$ using microplate spectrometer.

Figure 3. CD spectra of intermolecular SS mutants. The intermolecular SS mutants were dialyzed against $50 \mathrm{mM} \mathrm{KH}_{2} \mathrm{PO}_{4}$ buffer, $\mathrm{pH} 6.5$, and prepared at a concentration of $0.2 \mathrm{mg} / \mathrm{ml}$. Then, the CD spectra were collected on a spectropolarimeter.

Figure 4. DSC measurements. Thermograms of Mut3, Mut4, Mut5, Mut6, Mut7, and Mut8 monitored from 25 and $90{ }^{\circ} \mathrm{C}$ at a scan rate of $60^{\circ} \mathrm{C} / \mathrm{h}$. The protein solutions were prepared at concentration of $0.2 \mathrm{mg} / \mathrm{ml}$ in $50 \mathrm{mM}$ 
$\mathrm{KH}_{2} \mathrm{PO}_{4}$ buffer, $\mathrm{pH} 6.5$.

Figure 5. SDS-PAGE analysis of refolded Fab. The samples of (a) refolded WT and (b) refolded Mut 1 analyzed by 12\% SDS-PAGE under non-reduced condition. The gels were stained with SimplyBlue safe stain (Invitrogen) and were then imaged using iBright FL1000 (Thermo Fisher Scientific).

Figure 6. Mutation site of intermolecular SS mutants. Three-dimensional structure of adalimumab Fab (protein data bank number : 4NYL). The mutated amino acids are shown as sticks. 
Table. 1

Mutation site of Fab

\begin{tabular}{lcc}
\hline mutant & Hchain site & Lchain site \\
\hline Mut1 & H172C & S174C \\
Mut2 & S187C & S176C \\
Mut3 & F130C & Q124C \\
Mut4 & F174C & S176C \\
Mut5 & V177C & Q160C \\
Mut6 & F174C & S162C \\
Mut7 & F130C & S121C \\
Mut8 & A145C & F116C \\
Mut9 & L132C & F118C \\
\hline
\end{tabular}


Figures

Fig. 1

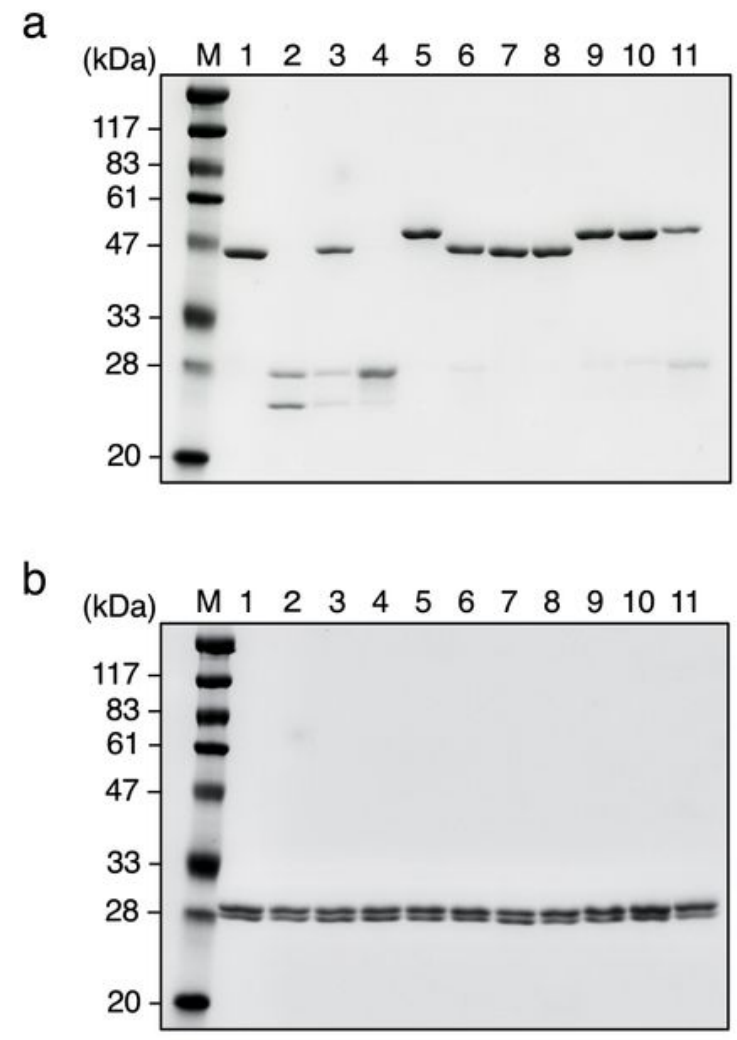

Figure 1

SDS-PAGE analysis of purified intermolecular SS mutants. The samples were analyzed by $12 \%$ SDS-PAGE under (a) non-reduced condition and (b) reduced condition. Lane M: protein markers. Lane 1: WT, Lane 2: $\Delta$ SSWT, Lane 3: Mut1, Lane 4: Mut2, Lane 5: Mut3, Lane 6: Mut4, Lane 7: Mut5, Lane 8: Mut6, Lane 9: 
Mut7, Lane 10: Mut8, Lane 11: Mut9. The gels were stained with SimplyBlue safe stain (Invitrogen) and were then imaged using iBright FL1000 (Thermo Fisher Scientific).

Fig. 2

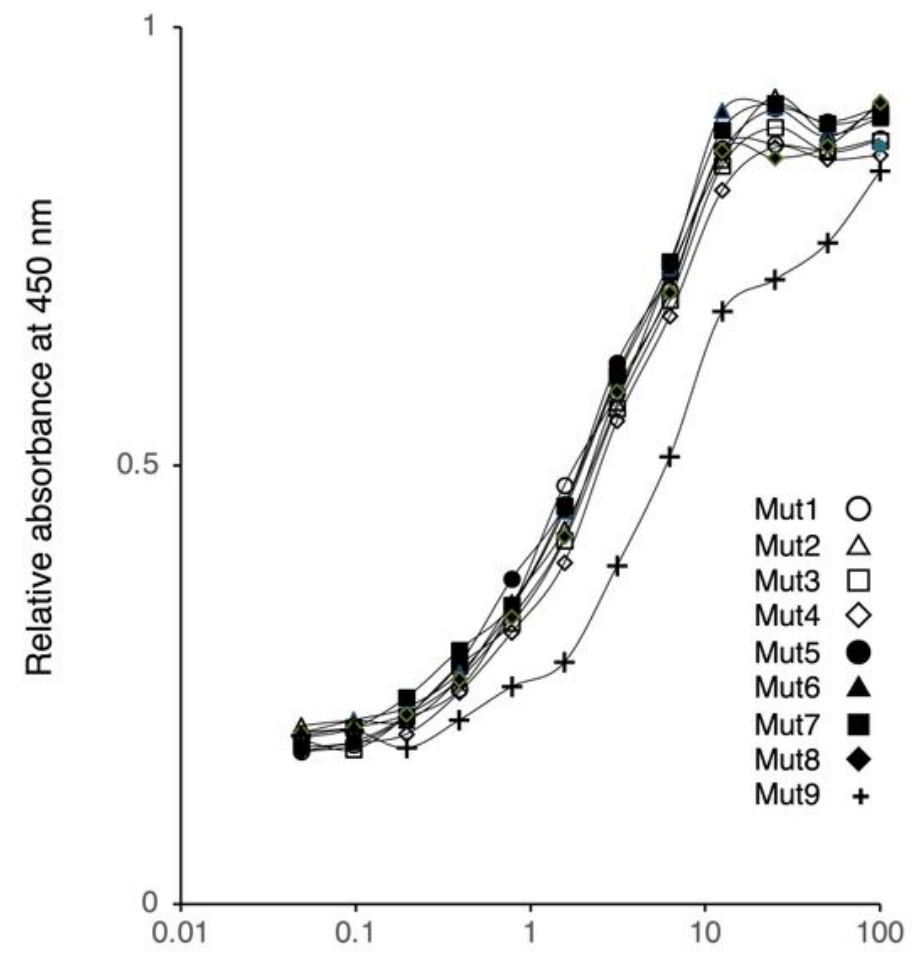

$\mathrm{Fab}(\mathrm{nM})$

\section{Figure 2}

The antigen-binding activity of intermolecular SS mutants. The ELISA plate was coated with TNFa, followed by addition of intermolecular SS mutants at various concentrations. Anti-human Fab conjugated 
with horseradish peroxidase polyclonal antibody was used for evaluating the antigen-binding activity. The absorbance was measured at $450 \mathrm{~nm}$ using microplate spectrometer

Fig. 3

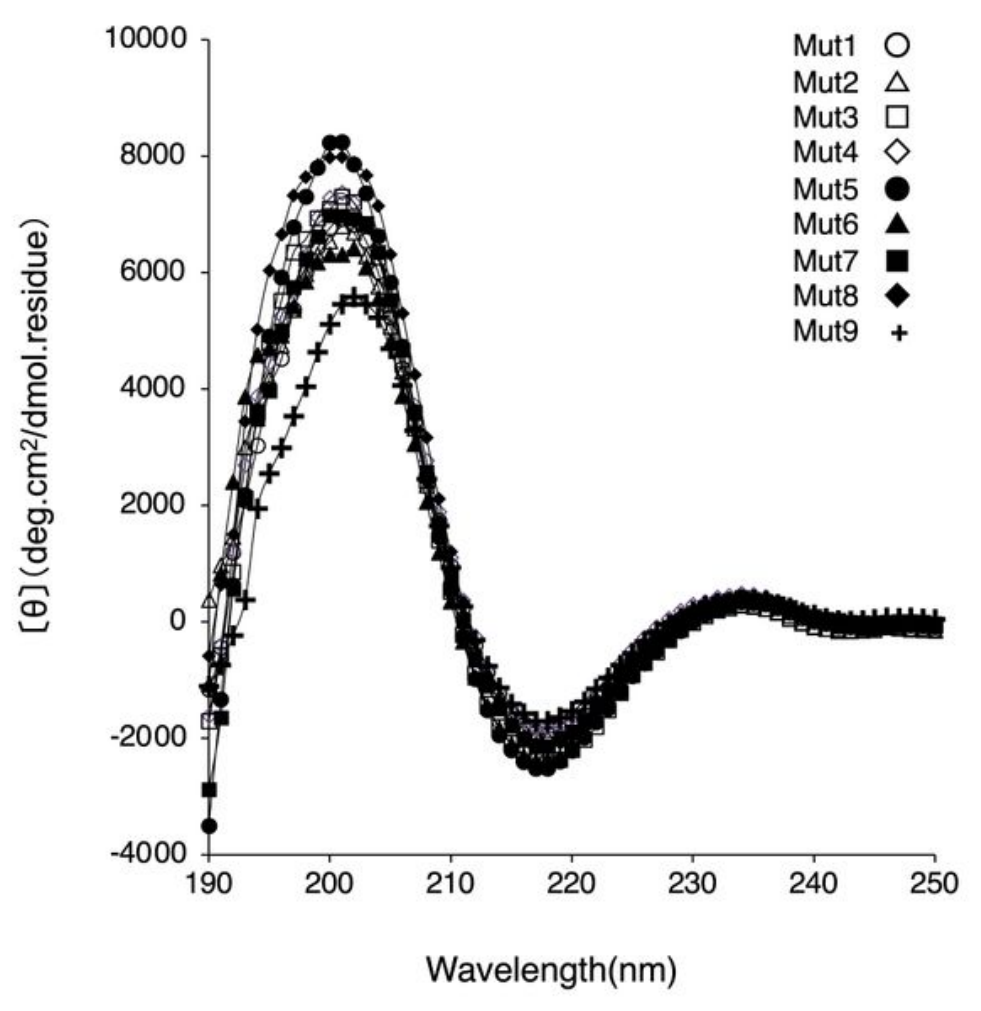

Figure 3

CD spectra of intermolecular SS mutants. The intermolecular SS mutants were dialyzed against $50 \mathrm{mM}$ $\mathrm{KH} 2 \mathrm{PO} 4$ buffer, $\mathrm{pH} 6.5$, and prepared at a concentration of $0.2 \mathrm{mg} / \mathrm{ml}$. Then, the $\mathrm{CD}$ spectra were collected on a spectropolarimeter 
Fig. 4
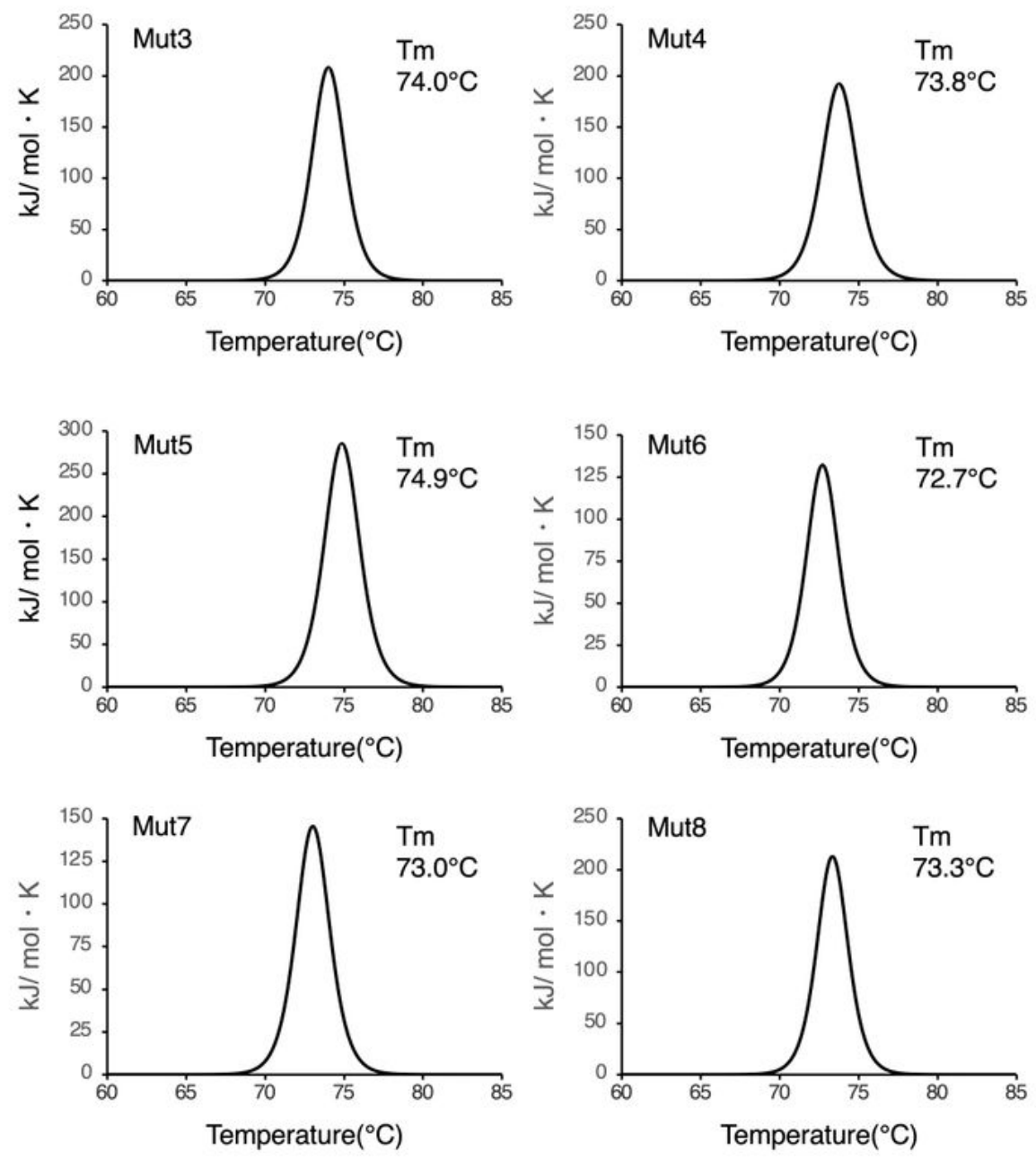

\section{Figure 4}

DSC measurements. Thermograms of Mut3, Mut4, Mut5, Mut6, Mut7, and Mut8 monitored from 25 and $90^{\circ} \mathrm{C}$ at a scan rate of $60^{\circ} \mathrm{C} / \mathrm{h}$. The protein solutions were prepared at concentration of $0.2 \mathrm{mg} / \mathrm{ml}$ in 50 $\mathrm{mM} \mathrm{KH} 2 \mathrm{PO} 4$ buffer, $\mathrm{pH} 6.5$ 
Fig. 5
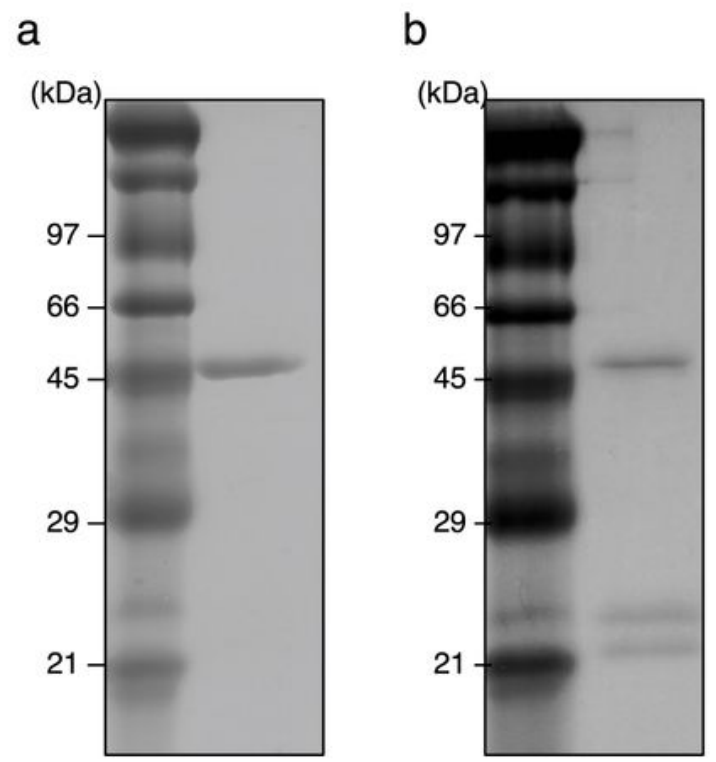

\section{Figure 5}

SDS-PAGE analysis of refolded Fab. The samples of (a) refolded WT and (b) refolded Mut1 analyzed by $12 \%$ SDS-PAGE under non-reduced condition. The gels were stained with SimplyBlue safe stain (Invitrogen) and were then imaged using iBright FL1000 (Thermo Fisher Scientific). 
Fig. 6
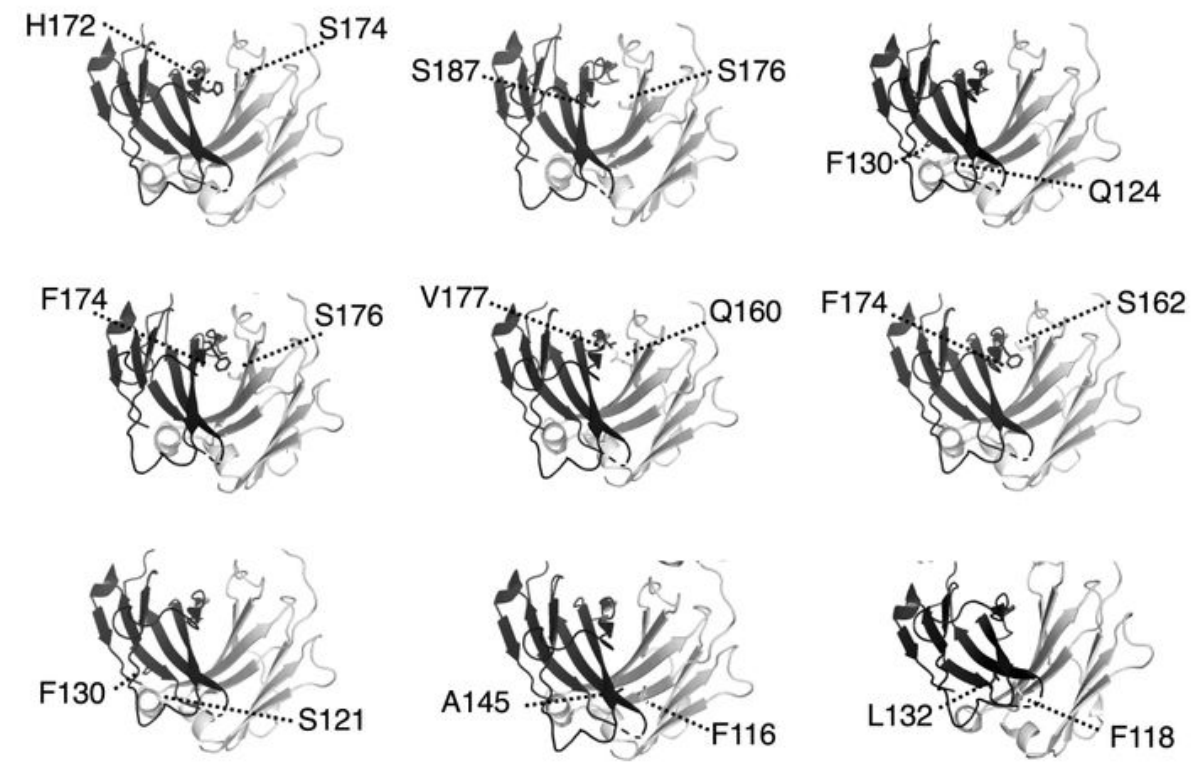

\section{Figure 6}

Mutation site of intermolecular SS mutants. Three-dimensional structure of adalimumab Fab (protein data bank number : $4 \mathrm{NYL}$ ). The mutated amino acids are shown as sticks.

\section{Supplementary Files}


This is a list of supplementary files associated with this preprint. Click to download.

- SupplementaryFigure.pdf 